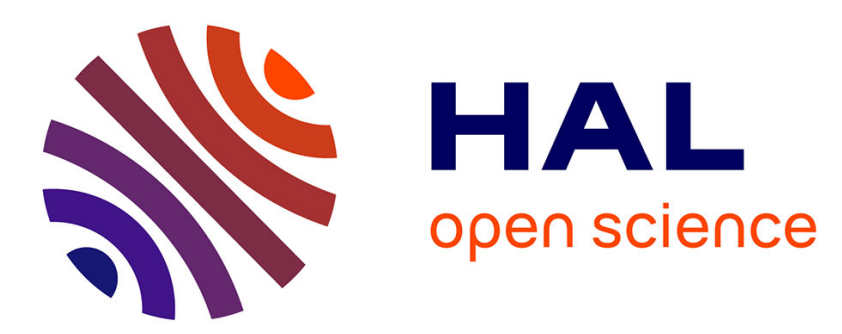

\title{
Mesure quantitative de la porosité résiduelle des ciments chirurgicaux par ultrasons de haute fréquence
}

\author{
Philippe Pernod, Christophe Delebarre, P. Hernigou, H. Frances
}

\section{To cite this version:}

Philippe Pernod, Christophe Delebarre, P. Hernigou, H. Frances. Mesure quantitative de la porosité résiduelle des ciments chirurgicaux par ultrasons de haute fréquence. Journal de Physique IV Proceedings, 1994, 04 (C5), pp.C5-1239-C5-1242. 10.1051/jp4:19945274 . jpa-00252965

\section{HAL Id: jpa-00252965 https://hal.science/jpa-00252965}

Submitted on 1 Jan 1994

HAL is a multi-disciplinary open access archive for the deposit and dissemination of scientific research documents, whether they are published or not. The documents may come from teaching and research institutions in France or abroad, or from public or private research centers.
L'archive ouverte pluridisciplinaire HAL, est destinée au dépôt et à la diffusion de documents scientifiques de niveau recherche, publiés ou non, émanant des établissements d'enseignement et de recherche français ou étrangers, des laboratoires publics ou privés. 


\title{
Mesure quantitative de la porosité résiduelle des ciments chirurgicaux par ultrasons de haute fréquence
}

\author{
P. PERNOD, C. DELEBARRE, P. HERNIGOU* et H. FRANCES
}

\author{
Institut d'Electronique et de Micro-électronique du Nord, Département Opto-Acousto-Electronique, \\ IEMN-DOAE, UMR 9929 du CNRS, Ecole Centrale de Lille, BP. 48, 59651 Villeneuve d'Ascq cedex, \\ France \\ ${ }^{*}$ Hopital Universitaire Henri Mondor, Service d'Orthopédie, 51 Av. Mal de Lattre de Tassigny, \\ 94000 Créteil, France
}

\begin{abstract}
A high frequency ultrasonic quantitative characterization of the porosity of biological cements for the fixation of prosthetic components has been realised. The images were acquired with a $\mathrm{C}-\mathrm{Scan}$ system using a focused $50 \mathrm{MHz}$ transducer. The images were treated by a histogram processing technique associated with a segmentation algorithm. The use of this system has allowed us to demonstrate that a decrease in porosity could be obtained by a polymerization under a high power insonification at $500 \mathrm{kHz}$.
\end{abstract}

\section{INTRODUCTION}

Le descellement des prothèses articulaires cimentées est un problème majeur en orthopédie, la durée de tenue des implants étant en moyenne limitée à 10 ans. Les origines de ces descellements viennent soit d'une maladie de l'os, soit de l'apparition d'une défaillance mécanique du ciment de fixation (PMMA), ou de l'une des interfaces ciment-prothèse, ou ciment-os. Dans ces derniers cas, la porosité contenue dans les ciments, due au processus particulier d'autopolymérisation à température / pression ambiantes et mélange manuel, semble jouer un rôle important.

Les techniques actuellement utilisées pour l'analyse de la porosité des ciments de prothèses sont limitées à la microscopie optique et la microscopic électronique à balayage. Dans les deux cas l'analyse en profondeur dans le matériau, et l'observation des interfaces, nécessitent une coupe des échantillons au niveau désiré, et les images obtenues se limitent aux informations contenues dans ce plan. La microscopie acoustique à balayage semble un moyen alternatif intéressant pour l'étude volumique non destructive de la porosité des ciments et l'étude des interfaces.

Après une description des paramètres choisis pour le système d'imagerie, nous présentons dans cet article les résultats obtenus pour des échantillons préparés dans les conditions standards préconisées par le fabricant, puis polymérisés sous insonification d'ultrasons de haute puissance et sous vide. La technique d'imagerie nous a permis de mettre en évidence la réduction de porosité ainsi obtenue.

\section{MATERIEL ET METHODE}

\subsection{Présentation du ciment}

Dans une étude préliminaire par microscopie électronique à balayage que nous avions effectué sur des échantillons de ciments (Cerafix Genta) réalisés en laboratoire [1], nous avions montré l'existence, outre de zircone et autres additifs, de deux types de porosités (figure 1) :

- des porosités de $5 \mu \mathrm{m}$ à $50 \mu \mathrm{m}$ de diamètre, de forme relativement bien sphérique, provenant probablement de la vaporisation du monomère durant la polymérisation ou de gaz ou d'air emprisonnés durant le mélange manuel,

- de porosités beaucoup plus grandes (diamètre $200 \mu \mathrm{m}$ à $300 \mu \mathrm{m}$ ) de forme irrégulière présentant des angles 


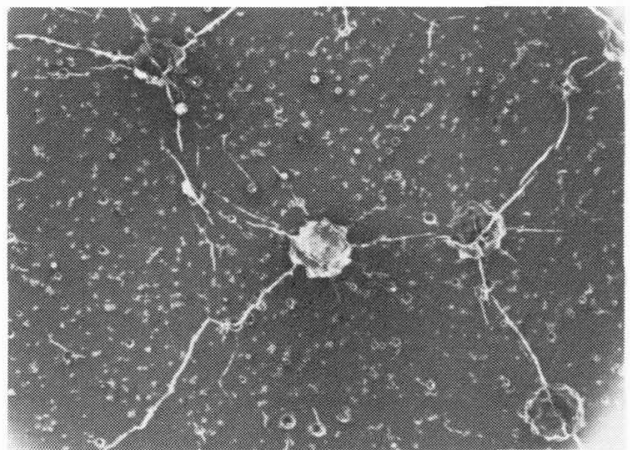

Figure 1 : Analyse par microscopie électronique à balayage d'un ciment biologique Cérafix.

vifs dans leur surface, constituant des zones privilégiées d'amorçages de fỉssures. Ce sont surtout ces dernières porosités qui nous sont apparues comme néfastes pour la tenue mécanique du ciment.

Dans cette étude nous avons réalisé deux types d'échantillons de dimensions $7 \times 1 \times 0,3 \mathrm{~cm}^{3}$ : une première catégorie réalisée dans les conditions standards préconisées par le fabricant ; une seconde, la polymérisation s'effectuant sous insonification d'ultrasons de fréquence $500 \mathrm{kHz}$, afin d'essayer de réduire la porosité.

\subsection{Dispositif expérimental}

Les images acoustiques ont été obtenues à l'aide d'un système C-Scan (figure 2) : Un transducteur Biosonic IF de $50 \mathrm{MHz}$ de fréquence centrale, fonctionnant sur le mode longitudinal, de $2 \mathrm{~mm}$ de rayon de courbure, $\pm 7 \%$ d'ouverture angulaire, et de $100 \%$ de bande passante relative $(-3 \mathrm{~dB})$, a été utilisé. La distance focale dans le matériau choisi était de $0,15 \mathrm{~mm}$ et la résolution latérale de $90 \mu \mathrm{m}$, cette dernière étant suffisante pour la détection des grosses porosités des ciments.

L'électronique (ER/100) est capable de délivrer une impulsion de $-180 \mathrm{~V}$ dans une charge de 50 Ohms, le temps de chute de l'impulsion étant de $1,5 \mathrm{~ns}$, la largeur de bande en réception $(-3 \mathrm{~dB})$ s'étendant de $10 \mathrm{MHz}$ à $550 \mathrm{MHz}$, le gain étant réglé électroniquement de façon calibrée de 0 à $70 \mathrm{~dB}$ par bonds de $1 \mathrm{~dB}$. L'intérêt majeur de ce système réside dans le détecteur de pic qui permet la détection monocoup de l'amplitude de l'écho radiof réquence $300 \mathrm{MHz}$ dans une porte de largeur $20 \mathrm{~ns}$.

L'écho rétrodiffusé provenant de la tache focale est détecté et converti sur une échelle de 64 niveaux. Un histogramme de l'image (nombre de pixels dans chaque niveau de digitalisation de l'amplitude) fourni alors la distribution des niveaux dans l'image du matériau, ce qui permet la caractérisation locale de sa structure [2]. Le pas de déplacement mécanique utilisé $(10 \mu \mathrm{m})$ est toujours resté inférieur à la résolution latérale $(90 \mu \mathrm{m})$. Par ailleurs un processus de calibration utilisant un échantillon test a été utilisé avant chaque acquisition afin de ré-
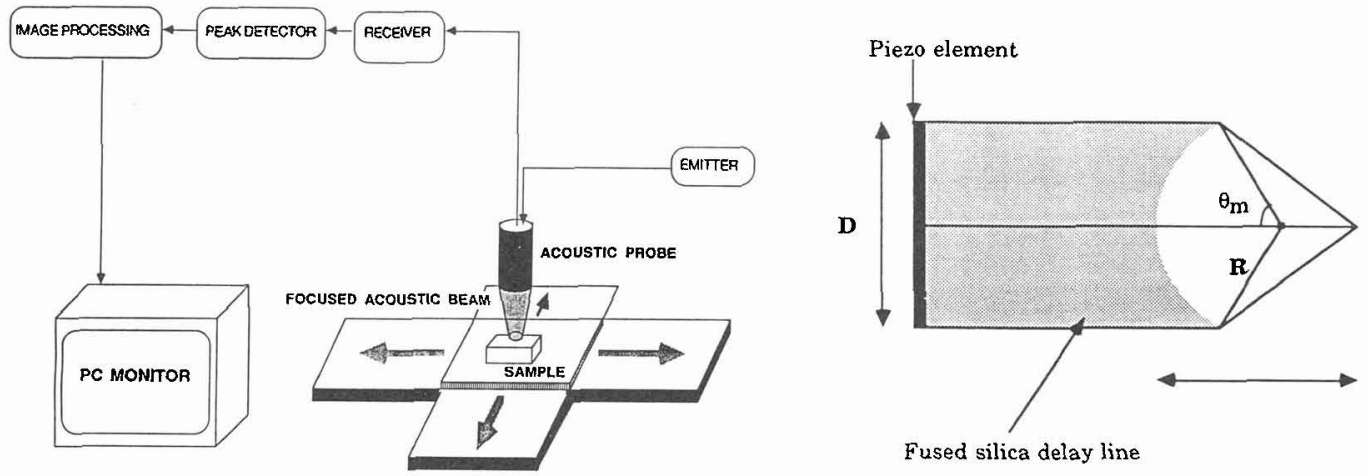

Figure 2 : Schéma de principe du dispositif C-Scan ; description du tranducteur $50 \mathrm{MHz}$. 
gler le gain du système à une valeur correcte et s'affranchir en particulier de l'influence de la température du milieu de couplage.

\section{RESULTATS}

Une série de 9 images situées en différentes zones des deux types d'échantillons produits (conditions standards \& sous ultrasons) a été réalisée. Les figures 3a,b présentent une image caractéristique de chacun des deux échantillons. La porosité qui se traduit par des éléments réflecteurs fortement échogènes apparaît sur les images comme des taches blanches, beaucoup plus nombreuses sur la préparation dans les conditions standards que sur les échantillons polymérisés sous uitrasons.

Afin de caractériser la porosité observée sur les images, deux paramètres des histogrammes ont été calculés : la valeur moyenne $\mathrm{m}$, et la déviation standard $\mathrm{s}$ définies respectivement par :

$$
m=\sum_{i} \frac{x_{i} \cdot n_{i}}{N} \quad s=\left[\sum_{i}\left[\frac{x_{i}^{2} \cdot n_{i}}{N-m^{2}}\right]\right]^{\frac{1}{2}}
$$

Le graphe représentant la valeur moyenne en fonction de la déviation standard des échantillons est présenté figure 4 : les échantillons standards sont bien différenciables des échantillons polymérisés sous ultrasons de puissance, les valeurs de moyenne et déviation standards étant toutes deux plus faibles dans ce dernier cas. La quantification de la porosité de l'image a été réalisée par l'utilisation de l'algorithme de segmentation de Bayes [2], méthode statistique consistant à associer à chaque pixel $\left(\mathrm{x}_{\mathrm{i}}\right)$ la classe $\left(\mathrm{w}_{\mathrm{k}}\right)$ pour laquelle la probabilité conditionnelle d'appartenance du niveau $\mathrm{x}_{\mathrm{i}}$ à la classe $\mathrm{w}_{\mathrm{k}} \mathrm{p}\left[\mathrm{w}_{\mathrm{k}} / \mathrm{x}_{\mathrm{i}}\right]$ est la plus grande.

(a)

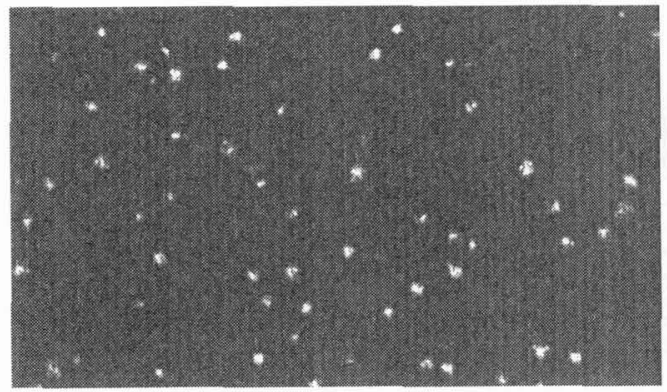

(b)

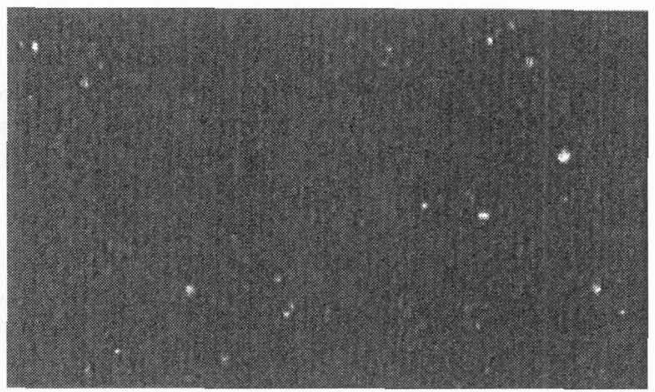

Figure 3 : Images ultrasonores d'un échantillon de ciment préparé dans les conditions standards préconisées par le fabricant (a), et sous insonification $500 \mathrm{kHz}$ haute puissance (b).

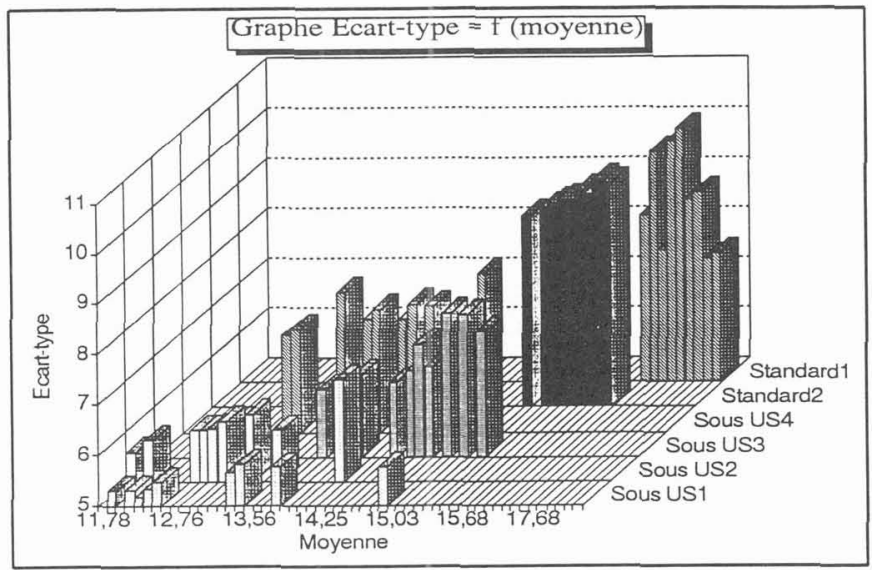

Figure 4 : Déviation standard et valeur moyenne de l'histogramme 


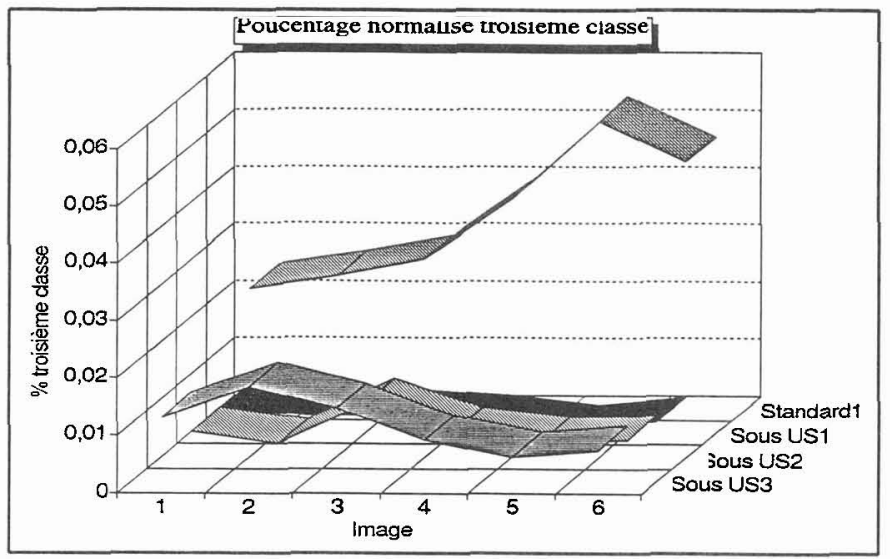

Figure 5 : Pourcentages de la troisième classe : comparaison échantillon standard / échantillon polymérisé sous ultrasons.

Les images ont été segmentées en 3 classes, notre intérêt se portant sur la classe contenant les niveaux de réflexion Les images ont été segmentées en 3 classes, notre intérêt se portant sur la classe contenant les niveaux de réflexion les plus élevés, associés à la porosité. La figure 5 montre une bonne séparation entre les ciments préparés dans les conditions standards et sous ultrasons. La porosité intrinsèque de $6 \%$ pour le premier type d'échantillon a été réduite à $1 \%$ pour l'utilisation des ultrasons de puissance.

\section{CONCLUSION}

L'imagerie acoustique haute fréquence associée aux techniques d'histogrammes et de segmentation d'images nous a permis de réaliser une caractérisation quantitative de la porosité des ciments biologiques. Grâce à cette technique nous avons montré que la porosité pouvait être réduite d'un facteur 6 , en réalisant la polymérisation sous ultrasons de puissance.

\section{REFERENCES}

[1] P. Hernigou, P. Pernod, J.Y. Dauphin, "Etude microstructurale du ciment et des interfaces os-ciment-prothèses", 7th International Symposium organized by the research group on Biomaterials and Grafts, Ile de Bendor, June 1991.

[2] C. Delebarre et al., "Quantitative characterization of metal matrix composites using histogram processing techniques", NDT and E International, Vol. 25 n4/5, pp. 195-200, 1992.

[3] C. Banga, F. Ghorbel, W. Pieczinski, "Unsupervised Bayesian classifier applied to the segmentation of retina image", IEEE-EMBS proceeding or the 14 th Annual International Conference PP 1847-1848, Oct. 1992. 